\title{
Article
}

\section{Zebrafish-Based Discovery of Antiseizure Compounds from the Red Sea: Pseurotin A2 and Azaspirofuran A}

Copmans, Daniëlle, Rateb, Mostafa, Tabudravu, Jioji, Pérez-Bonilla, Mercedes, Dirkx, Nina, Vallorani, Riccardo, Diaz, Caridad, Pérez del Palacio, José, Smith, Alan J., Ebel, Rainer, Reyes, Fernando, Jaspars, Marcel and de Witte, Peter A. M.

Available at https://clok.uclan.ac.uk/22287/

Copmans, Daniëlle, Rateb, Mostafa, Tabudravu, Jioji orcid iconORCID: 00000002-6930-6572, Pérez-Bonilla, Mercedes, Dirkx, Nina, Vallorani, Riccardo, Diaz, Caridad, Pérez del Palacio, José, Smith, Alan J. et al (2018) ZebrafishBased Discovery of Antiseizure Compounds from the Red Sea: Pseurotin A2 and Azaspirofuran A. ACS Chemical Neuroscience, 9 (7). pp. 1652-1662. ISSN 1948-7193

It is advisable to refer to the publisher's version if you intend to cite from the work. http://dx.doi.org/10.1021/acschemneuro.8b00060

For more information about UCLan's research in this area go to http://www.uclan.ac.uk/researchgroups/ and search for <name of research Group>.

For information about Research generally at UCLan please go to http://www.uclan.ac.uk/research/

All outputs in CLoK are protected by Intellectual Property Rights law, including Copyright law. Copyright, IPR and Moral Rights for the works on this site are retained by the individual authors and/or other copyright owners. Terms and conditions for use of this material are defined in the policies page. 


\section{Zebrafish-based discovery of antiseizure compounds from the Red Sea: pseurotin $\mathrm{A}_{2}$ and azaspirofuran $\mathrm{A}$}

Daniëlle Copmans ${ }^{1, \#}$, Mostafa Rateb ${ }^{2,3,4, \#}$, Jioji N. Tabudravu ${ }^{2,5}$, Mercedes Pérez-Bonilla ${ }^{6}$, Nina Dirkx ${ }^{1}$, Riccardo Vallorani ${ }^{1}$, Caridad Diaz ${ }^{6}$, José Pérez del Palacio ${ }^{6}$, Alan J. Smith ${ }^{2}$, Rainer Ebel $^{2}$, Fernando Reyes ${ }^{6}$, Marcel Jaspars ${ }^{2, *}$, Peter A. M. de Witte ${ }^{1, *}$

${ }^{1}$ Laboratory for Molecular Biodiscovery, Department of Pharmaceutical and Pharmacological Sciences, KU Leuven - University of Leuven, 3000 Leuven, Belgium

${ }^{2}$ Marine Biodiscovery Centre, Department of Chemistry, University of Aberdeen, Aberdeen AB24 3UE, Scotland, UK

${ }^{3}$ Faculty of Pharmacy, Pharmacognosy Department, Beni-Suef University, Beni-Suef 62513, Egypt

${ }^{4}$ Current affiliation: School of Science \& Sport, University of the West of Scotland, Paisley PA1 2BE, Scotland, UK

${ }^{5}$ Current affiliation: School of Forensics and Applied Sciences, Faculty of Science and Technology, University of Central Lancashire, Preston PR1 2HE, UK.

${ }^{6}$ Fundación MEDINA, Centro de Excelencia en Investigación de Medicamentos Innovadores en Andalucía, Parque Tecnológico de Ciencias de la Salud, E-18016 Granada, Spain.

${ }^{\#}$ Authors contributed equally.

*Corresponding authors:

Prof. Dr. Peter A. M. de Witte, Laboratory for Molecular Biodiscovery, Department of Pharmaceutical and Pharmacological Sciences, KU Leuven, Herestraat 49 box 824, 3000 Leuven, Belgium. E-mail: peter.dewitte@kuleuven.be

Prof. Dr. Marcel Jaspars, Marine Biodiscovery Centre, Department of Chemistry, University of Aberdeen, Aberdeen AB24 3UE, Scotland, UK. E-mail: m.jaspars@abdn.ac.uk 


\begin{abstract}
In search for novel antiseizure drugs (ASDs), the European FP7-funded PharmaSea project used zebrafish embryos and larvae as a drug discovery platform to screen marine natural products to identify promising antiseizure hits in vivo for further development. Within the framework of this project, 7 known hetero-spirocyclic $\gamma$-lactams, namely pseurotin A, pseurotin $\mathrm{A}_{2}$, pseurotin F1, 11-O-methylpseurotin A, pseurotin D, azaspirofuran A, and azaspirofuran $\mathrm{B}$, were isolated from the bioactive marine fungus Aspergillus fumigatus, and their antiseizure activity was evaluated in the larval zebrafish pentylenetetrazole (PTZ) seizure model. Pseurotin $\mathrm{A}_{2}$ and azaspirofuran A were identified as antiseizure hits, while their close chemical analogues were inactive. Besides, electrophysiological analysis from the zebrafish midbrain demonstrated that pseurotin $\mathrm{A}_{2}$ and azaspirofuran A also ameliorate PTZ-induced epileptiform discharges. Next, to determine whether these findings translate to mammalians, both compounds were analyzed in the mouse $6-\mathrm{Hz}(44 \mathrm{~mA})$ psychomotor seizure model. They lowered the seizure duration dose-dependently, thereby confirming their antiseizure properties and suggesting activity against drug-resistant seizures. Finally, in a thorough ADMET assessment, pseurotin $\mathrm{A}_{2}$ and azaspirofuran A were found to be drug-like. Based on the prominent antiseizure activity in both species and the drug-likeness, we propose pseurotin $\mathrm{A}_{2}$ and azaspirofuran $\mathrm{A}$ as lead compounds that are worth further investigation for the treatment of epileptic seizures. This study not only provides the first evidence of antiseizure activity of pseurotins and azaspirofurans, but also demonstrates the value of the zebrafish model in (marine) natural product drug discovery in general, and for ASD discovery in particular.
\end{abstract}

\title{
KEYWORDS
}

epilepsy, antiseizure drug discovery, marine drug discovery, pseurotin $\mathrm{A}_{2}$, azaspirofuran A, Aspergillus fumigatus 


\section{INTRODUCTION}

Epilepsy is one of the most common neurological conditions, affecting more than 70 million people of all ages with no geographical, social, or racial boundaries. ${ }^{1-3}$ It is a disease of the brain that is characterized by spontaneous recurrent unprovoked seizures. ${ }^{4}$ Despite an exponential growth of marketed antiseizure drugs (ASDs) over the past 25 years, seizures remain uncontrolled in one third of the patients due to drug resistance. ${ }^{5,6}$ As uncontrolled epilepsy is associated with increased physical and physiological comorbidities and increased risk of sudden unexplained death, there is a substantial burden on the patients, their caretakers and society. ${ }^{6}$ Hence, more efficacious ASDs that can treat patients with drug-resistant seizures are sorely needed. ${ }^{7}$

The EU PharmaSea project, funded by the Seventh Framework Programme (FP7), is an SMEacademia-driven project of 24 partners that was initiated in 2012 to discover novel antibiotic, anti-inflammatory and neuroactive compounds from marine microorganisms isolated from some of the deepest, coldest, and hottest places on the planet. ${ }^{8}$ Neuroactive drug discovery focused on the identification of compounds with the potential to treat epilepsy. To the best of our knowledge, we have been the first to perform systematic large-scale screening of marinederived natural products for antiseizure drug candidates. Marine species offer an attractive source for drug discovery because they produce potent, selective, and structurally novel bioactive secondary metabolites to defend themselves, to locate mates, and to out-compete competitors for limited resources. ${ }^{8-10}$ Many of these do not have a terrestrial analogue and are thus unique in terms of chemical structure and bioactivity. ${ }^{8,9}$ Among them are marine-derived natural products that can alter mammalian neurological activity. Classical examples are tetrodotoxins, saxitoxins, kainic and domoic acids, cone snail venom peptides, and sea anemone toxins. ${ }^{10}$ Nevertheless, marine natural products are highly underutilized in drug discovery. ${ }^{11} \mathrm{~A}$ main reason is the lack of systematic analysis in high-content bioassays given the often limited quantities of marine natural products available for screening purposes. ${ }^{11}$

The zebrafish model is suitable for large-scale screening and captures the complexity of a whole body organism, including the central nervous system. As a vertebrate, zebrafish are highly similar to humans due to a high genetic, physiological and pharmacological conservation. ${ }^{12-14}$ Moreover, given the small size of embryos and larvae, they fit in wells of microtiter plates and hence are suitable for medium to high-throughput testing. ${ }^{13,14}$ Given the low volumes used in 96- and 384-well plates, zebrafish larvae only require small amounts of sample in the low microgram range when added to their swimming water and even less when administered by 
injection. This property is of particular interest for marine natural product drug discovery, where material is often scarce. ${ }^{11}$ Within the PharmaSea project, marine samples were screened in 96-well plate format using only $10 \mu \mathrm{g}$ per well per screening round. From the established larval zebrafish seizure and epilepsy models ${ }^{15}$, the larval zebrafish pentylenetetrazole (PTZ) seizure model was chosen for drug discovery because: 1) the model has been extensively characterized in terms of behavioral and non-behavioral seizure markers ${ }^{16,17}$, 2) it has been pharmacologically characterized with ASDs on the market ${ }^{16-18}$, 3) results translate well to rodent models ${ }^{17}, 19,20$, and 4) the behavioral assay is suitable for high-throughput screening seizures can easily and rapidly be induced by a single administration of the convulsant drug to the larva's aqueous environment ${ }^{16}$ and can be quantified by video recording ${ }^{16-18}$.

Within the framework of the PharmaSea project, 5 pseurotins (pseurotin A, pseurotin $A_{2}$, pseurotin $\mathrm{F} 1,11-O$-methylpseurotin $\mathrm{A}$, and pseurotin $\mathrm{D}$ ) and 2 azaspirofurans (azaspirofuran $\mathrm{A}$ and B) were isolated from extracts of the bioactive marine fungus Aspergillus fumigatus, which was collected from a Red Sea sediment in Hurghada, Egypt. Interestingly, these compounds did not demonstrate antibacterial activity or cytotoxicity in contrast to other constituents from the crude extract ${ }^{21}$ and were readily available for further biological evaluation. Pseurotins are a family of fungal secondary metabolites that have not been well studied so far, except for pseurotin A that was found to exhibit a range of bioactivities at moderate to high test concentrations (up to $50 \mu \mathrm{g} / \mathrm{mL}$ ). ${ }^{22-31}$ Azaspirofurans are chemically very similar to pseurotins, but feature an ethyl furan ring instead of a vicinal diol (Figure 1). Little is known about the bioactivities of azaspirofurans as they were identified only recently. ${ }^{32}$ So far, azaspirofuran A was observed to specifically inhibit the proliferation of the A459 cancer cell line. ${ }^{32,33}$

All compounds were investigated for antiseizure activity in the larval zebrafish PTZ seizure model. Interestingly, despite close structural similarities, only pseurotin $\mathrm{A}_{2}$ and azaspirofuran A ameliorated PTZ-induced seizures, suggesting a highly specific interaction. Besides, electrophysiological analysis from the zebrafish midbrain demonstrated that pseurotin $\mathrm{A}_{2}$ and azaspirofuran A also significantly lowered PTZ-induced epileptiform discharges. Next, the antiseizure activity of pseurotin $\mathrm{A}_{2}$ and azaspirofuran $\mathrm{A}$ was demonstrated in the mouse $6-\mathrm{Hz}$ (44 mA) psychomotor seizure model. These results not only confirmed the translation of findings from zebrafish larvae to mice but also suggested that pseurotin $\mathrm{A}_{2}$ and azaspirofuran A are effective against drug-resistant focal seizures. Finally, ADMET profiling showed that both compounds are drug-like. Based on the prominent antiseizure activity and the promising 
ADMET characteristics, we propose pseurotin $\mathrm{A}_{2}$ and azaspirofuran $\mathrm{A}$ as lead compounds that are worth further investigation for the treatment of seizures.

\section{RESULTS AND DISCUSSION}

Isolation and structural elucidation of compounds from the bioactive marine fungus Aspergillus fumigatus

The marine fungal isolate MR2012 used in this study was isolated from a Red Sea sediment in Hurghada, Egypt and taxonomically identified on a molecular basis as Aspergillus fumigatus ${ }^{21}$. Compounds pseurotin $\mathrm{A}^{34}$, pseurotin $\mathrm{A}_{2}{ }^{27}$, pseurotin $\mathrm{F}^{35}$, $11-O$-methylpseurotin $\mathrm{A}^{34}$, pseurotin $\mathrm{D}^{36}$, and azaspirofurans $\mathrm{A}$ and $\mathrm{B}^{32}$, all known hetero-spirocyclic $\gamma$-lactams, were isolated from the $\mathrm{CH}_{2} \mathrm{Cl}_{2}$ fraction of the fungal fermentation ${ }^{21,37}$ (Figure 1). Their structures were confirmed by HRESIMS analysis and by comparing the 1D and 2D NMR spectra and optical rotation with literature data as indicated (see supporting information for their 1D NMR data).

\section{Azaspirofuran A and pseurotin $A_{2}$ ameliorate seizures in the zebrafish PTZ seizure model}

To investigate whether the isolated compounds display antiseizure activity, they were tested in the larval zebrafish PTZ seizure model both after a short (2 hours (h)) and long (18 h) incubation time at their maximum tolerated concentration (MTC) (Table 1). The MTC was defined as the highest concentration at which no larvae died nor showed signs of toxicity or locomotor impairment in comparison to vehicle (VHC)-treated control larvae. In case no MTC was reached, $100 \mu \mathrm{g} / \mathrm{mL}$ was used as the test concentration. In line with studies previously reported $^{16,17}$, addition of the $\mathrm{GABA}_{\mathrm{A}}$-receptor antagonist PTZ to the swimming water of 7-days post-fertilization (dpf) zebrafish larvae strongly elevated larval locomotion $(\mathrm{p} \leq 0.001)$ (Figure 2 and 3 ) as a result of induced seizures (or seizure-like behavior) that were recognized as typical high-speed swimming, whirlpool-like circling, clonus-like seizures, and loss of posture, as previously described ${ }^{16}$. Azaspirofuran $\mathrm{A}(\mathrm{p} \leq 0.01)$ and pseurotin $\mathrm{A}_{2}(\mathrm{p} \leq 0.001)$ significantly lowered PTZ-induced seizures of larvae after 2 and $18 \mathrm{~h}$ of compound exposure, respectively (Figure 2). Remarkably, none of the chemical analogues showed notable activities despite their close structural similarities. These results suggest that azaspirofuran $\mathrm{A}$ and pseurotin $\mathrm{A}_{2}$ specifically interact with their antiseizure target(s). In contrast to azaspirofuran $\mathrm{A}$, azaspirofuran B lacks the methoxyl group (Figure 1), which seems necessary for the activity 
against PTZ-induced seizures. It is unlikely that the activity of azaspirofuran A is due to an improved compound uptake as the $\log \mathrm{P}$ of azaspirofuran $\mathrm{A}$ and $\mathrm{B}$ is predicted to be 3.98 and 3.81 (see methods), respectively, and good compound bioavailability in zebrafish larvae is expected from a $\log \mathrm{P}$ of 1 onwards ${ }^{38}$. The close analogues pseurotin $\mathrm{A}$ and $\mathrm{A}_{2}$ are diastereomers with different configurations at C-8 and C-9 (Figure 1). ${ }^{27}$ Their structural differences do not affect uptake, which is also expected to be adequate as the compounds are predicted to have a $\log \mathrm{P}$ of 3.23 (see methods). So, likely the structural differences result in distinct pharmacological activities rather than in altered bioavailabilities. Nevertheless the pharmacokinetics of the individual compounds in zebrafish larvae are currently unknown, so it cannot be ruled out that the actual brain concentrations of azaspirofuran A and pseurotin $A_{2}$ are higher than those of their inactive analogues. Of note, like azaspirofuran A, pseurotin A, pseurotin $\mathrm{D}$, and 11-O-methylpseurotin A also possess the methoxyl group and are not active, which can be due to the absence of the ethyl furan ring. These observations suggest that the molecular target(s) of azaspirofuran $\mathrm{A}$ and pseurotin $\mathrm{A}_{2}$ are not necessarily the same. Interestingly, antiseizure activity has not yet been reported for azaspirofurans or pseurotins.

To investigate the concentration-response relationship, azaspirofuran A and pseurotin $\mathrm{A}_{2}$ were retested at their MTC, MTC/2, and MTC/4 (two-fold serial dilution) in the zebrafish PTZ seizure model at their optimal incubation time ( 2 and $18 \mathrm{~h}$, respectively) in three independent experiments (Figure 3). Azaspirofuran A lowered PTZ-induced seizure behavior to the same extent as before at the MTC over the 30-minute ( $\mathrm{min}$ ) recording period (Figure 3A). A more detailed analysis of the 5-min time intervals from the 30-min recording period revealed a significant reduction in the 10-30 min time window ( $\leq 0.001)$ (Figure 3B). No antiseizure activity was seen at lower concentrations. Pseurotin $\mathrm{A}_{2}$ showed concentration-dependent activity against PTZ-induced seizure behavior, both within the 30 -min recording period $(\mathrm{p} \leq$ 0.01 , Figure 3C) as during the 5-min time intervals ( $\mathrm{p} \leq 0.001$, Figure 3D).

\section{Azaspirofuran A and pseurotin $A_{2}$ ameliorate epileptiform brain activity in the zebrafish PTZ seizure model}

To determine whether azaspirofuran $\mathrm{A}$ and pseurotin $\mathrm{A}_{2}$ can ameliorate the PTZ-induced hyperexcitable state of the brain that is characterized by epileptiform discharges ${ }^{39}$, local field potential (LFP) recordings ${ }^{40}$ were non-invasively measured from the midbrain (optic tectum) of zebrafish larvae. To that end larvae were treated with either VHC or test compound (MTC 
and optimal incubation time was used) followed by a 15 min during exposure to PTZ or VHC prior to the electrophysiology measurements (Figure 4 and 5). A larva was considered to have epileptiform brain activity when at least 3 electrical discharges were seen in the 10-min recording period that fulfilled the pre-defined requirements of an epileptiform event (see methods). Pre-incubation with azaspirofuran A significantly reduced $(\mathrm{p} \leq 0.001)$ the percentage of larvae with PTZ-induced epileptiform activity with almost $60 \%$ in comparison to PTZtreated controls (Figure 4A). Larvae also showed significantly less epileptiform events ( $\mathrm{p} \leq$ 0.001) when pre-exposed to azaspirofuran A, resulting in a shorter cumulative duration of events $(\mathrm{p} \leq 0.001)$ over the 10 -min recording period (Figure 4B and C). Pseurotin $\mathrm{A}_{2}$ only nonsignificantly lowered the percentage of larvae with PTZ-induced epileptiform activity with 33 $\%$, in comparison to controls (Figure 4D). However, larvae did show significantly less epileptiform events $(p \leq 0.01)$, resulting in a shorter cumulative duration of events $(p \leq 0.05)$ over the 10-min recording period (Figure $4 \mathrm{E}$ and F). Thus, both compounds not only ameliorate PTZ-induced seizures but are likely to do so by lowering the PTZ-induced hyperexcitable state of the brain. Of note, azaspirofuran A or pseurotin $A_{2}$ did not induce abnormal electrical discharges in comparison to VHC-treated controls.

\section{Azaspirofuran A and pseurotin $A_{2}$ ameliorate focal seizures in the mouse 6-Hz (44 mA) psychomotor seizure model}

Although the zebrafish model has a high degree of genetic, physiological, and pharmacological conservation $^{13}$, it is more distinct from humans than rodents. Therefore, we were interested to see if the observed antiseizure properties of azaspirofuran A and pseurotin $A_{2}$ would translate to a rodent model. The mouse 6-Hz (44 mA) psychomotor seizure model was chosen because it can detect compounds with novel antiseizure mechanisms and with potential against drugresistant seizures. ${ }^{41,} 42$ In this model drug-resistant focal impaired awareness seizures ${ }^{43}$, previously referred to as complex partial or psychomotor seizures ${ }^{44}$, are induced by low frequency, long duration corneal electrical stimulation. Mice injected i.p. with VHC (30 min before electrical stimulation) had a mean $( \pm$ SD) seizure duration of 50 seconds $(s)( \pm 19 s)$ (Figure 6). In line with previous studies ${ }^{41,45}$, i.p. administration of the positive control valproate (300 mg/kg dose, $30 \mathrm{~min}$ before electrical stimulation) protected all mice against the induced seizures, significantly reducing the seizure duration to a mean of $4 \mathrm{~s}( \pm 6 \mathrm{~s})(\mathrm{p} \leq 0.001)$. In contrast, i.p. administration of the negative control phenytoin $(10 \mathrm{mg} / \mathrm{kg}$ dose, $120 \mathrm{~min}$ before electrical stimulation, as reported by Barton and colleagues ${ }^{41}$ ) did not significantly affect the 
seizure duration (mean duration of $30 \mathrm{~s}( \pm 13 \mathrm{~s})$ ) as previously published ${ }^{41,46,47}$. Administration of $40 \mathrm{mg} / \mathrm{kg}$ azaspirofuran A (i.p. injected, $30 \mathrm{~min}$ before electrical stimulation) significantly lowered the seizure duration to a mean of $24 \mathrm{~s}( \pm 18 \mathrm{~s})(\mathrm{p} \leq 0.05)$ in comparison to VHCcontrols. Administration of $40 \mathrm{mg} / \mathrm{kg}$ pseurotin A2 (i.p. injected, $30 \mathrm{~min}$ before electrical stimulation) significantly lowered the seizure duration to a mean of $26 \mathrm{~s}( \pm 18 \mathrm{~s})(\mathrm{p} \leq 0.05)$ in comparison to controls. Treatment with azaspirofuran A as well as with pseurotin $\mathrm{A}_{2}$ showed a trend for dose-dependent reduction in seizure duration. 20 and $10 \mathrm{mg} / \mathrm{kg}$ azaspirofuran A nonsignificantly lowered the seizure duration to a mean of 41 and $46 \mathrm{~s}$ ( \pm 15 and $20 \mathrm{~s}$ ), respectively. 10 and $2.5 \mathrm{mg} / \mathrm{kg}$ pseurotin $\mathrm{A}_{2}$ non-significantly lowered the seizure duration to a mean of 27 and $42 \mathrm{~s}$ ( \pm 26 and $11 \mathrm{~s}$ ), respectively. These data confirm the antiseizure properties of azaspirofuran $\mathrm{A}$ and pseurotin $\mathrm{A}_{2}$ in a standard mouse model of drug-resistant focal seizures. The identification and validation of these novel antiseizure hits thereby demonstrate the effectiveness of using the larval zebrafish model for ASD discovery. Besides, this study provides another example of the translation of results from zebrafish to rodent seizure models. Little is known about the molecular mechanisms underlying the bioactivities of azaspirofuran A and pseurotin $A_{2}$ in general. Therefore, it is difficult to speculate on the antiseizure drug targets that could be involved. Of note, pseurotin $\mathrm{A}_{2}$ was reported to exert anti-inflammatory activity, a promising mode of action that has been proposed for the development of innovative ASDs and AEDs as neuroinflammation is involved in the origin of seizures and epilepsy. ${ }^{29,48,}$

${ }^{49}$ In addition, pseurotin A is known to induce neuronal cell differentiation. ${ }^{22}$ This long-term effect is unlikely to be involved in the antiseizure actions of azaspirofuran $\mathrm{A}$ and pseurotin $\mathrm{A}_{2}$ in this study because of the short-term exposure ( 2 and $18 \mathrm{~h}$ of exposure, respectively), but it is of particular interest for AED development. Further research is needed to unravel the molecular mechanisms of azaspirofuran $\mathrm{A}$ and pseurotin $\mathrm{A}_{2}$ that are responsible for their antiseizure activity against PTZ-induced seizures in zebrafish and focal seizures in mice.

\section{ADMET profiling of azaspirofuran $A$ and pseurotin $\mathbf{A}_{2}$}

Finally, to define the drug-likeness of the antiseizure lead compounds, the ADMET profiles of azaspirofuran $\mathrm{A}$ and pseurotin $\mathrm{A}_{2}$ were elucidated using standard in vitro assays. The ADMET results are summarized in Table 2 . No notable cytotoxicity or cardiotoxicity was observed for either one of the compounds. Both compounds showed an acceptable solubility and azaspirofuran A also demonstrated a high permeability, a desired combination that is not common. Furthermore, azaspirofuran A only weakly inhibited the CYP2D6 and CYP2C9 
enzymes and did not affect the CYP3A4 enzyme, while pseurotin $\mathrm{A}_{2}$ did not inhibit any of the three types of CYP450 enzymes. Thus, azaspirofuran A and pseurotin $\mathrm{A}_{2}$ are unlikely to present drug-drug interactions. In addition, they are metabolically stable with a half-life ( $\left.\mathrm{t}_{1 / 2}\right)$ of 33 and $>60$ min for azaspirofuran A and pseurotin A2, respectively. Finally, azaspirofuran A showed a high level of plasma protein binding (95\%), which is not optimal but can be addressed, and pseurotin $\mathrm{A}_{2}$ has a much lower plasma protein binding of only $37 \%$ with good recovery. Thus, azaspirofuran $\mathrm{A}$ and pseurotin $\mathrm{A}_{2}$ show promising ADMET characteristics and are therefore drug-like. Hence, we propose azaspirofuran A and pseurotin $\mathrm{A}_{2}$ as lead compounds worth further investigation for the treatment of epileptic seizures in general, and drug-resistant focal seizures in particular.

\section{CONCLUSIONS}

In this study, 7 known hetero-spirocyclic $\gamma$-lactams were isolated from the marine sedimentderived fungus Aspergillus fumigatus MR2012 and investigated for antiseizure activity in the larval zebrafish PTZ seizure model. Pseurotin A2 and azaspirofuran A were found to have promising antiseizure activity, not present in the other structural analogues tested and thus suggested to have a structure-specific interaction with, possibly new, antiseizure drug targets. The antiseizure activity of pseurotin $\mathrm{A}_{2}$ and azaspirofuran $\mathrm{A}$ translated to a mouse model of drug-resistant focal seizures and in addition, both compounds were observed to be drug-like. Based on the prominent antiseizure activity in zebrafish and mice and their drug-likeness, we propose pseurotin $\mathrm{A}_{2}$ and azaspirofuran $\mathrm{A}$ as lead compounds that are worth further investigation for the treatment of epileptic seizures. Thereby, this study provides the first evidence of antiseizure activity of pseurotins and azaspirofurans, and gives another example of the translation of results from zebrafish larvae to mice. Moreover, this study demonstrates the value of the zebrafish model in (marine) natural product drug discovery in general, and for ASD discovery in particular. A detailed structure-activity relationship investigation is needed to understand the structural necessities of pseurotins and azaspirofurans to exert their antiseizure action. Moreover, further research is needed to unravel the molecular mechanisms of pseurotin $\mathrm{A}_{2}$ and azaspirofuran $\mathrm{A}$ that are responsible for their antiseizure activity against PTZ-induced seizures in zebrafish and focal seizures in mice. 


\section{METHODS}

\section{Chemical experimental procedures}

NMR data were acquired on a Varian Inova $600 \mathrm{MHz}$ NMR spectrometer. High resolution mass spectrometric data were obtained using a Thermo LTQ Orbitrap coupled to an HPLC system (PDA detector, PDA autosampler, and pump). The following conditions were used: capillary voltage of $45 \mathrm{~V}$, capillary temperature of $260{ }^{\circ} \mathrm{C}$, auxiliary gas flow rate of 10-20 arbitrary units, sheath gas flow rate of 40-50 arbitrary units, spray voltage of $4.5 \mathrm{kV}$, and mass range of 100-2000 amu (maximal resolution of 30,000). For LC/MS, a $\mathrm{C}_{18}$ analytical HPLC column (5 $\mu \mathrm{m}, 4.6 \mathrm{~mm} \times 150 \mathrm{~mm}$ ) was used with a mobile phase of 0 to $100 \% \mathrm{MeOH}$ over $30 \mathrm{~min}$ at a flow rate of $1 \mathrm{~mL} \mathrm{~min}^{-1}$. Biotage Flash system SP1-XOB1, Charlottesville WA, USA was used for initial purification. Compound purification was conducted using Agilent 1200 HPLC system with a Waters Sunfire $C_{18}$ column $(5 \mu \mathrm{m}, 100 \AA, 10 \mathrm{~mm} \times 250 \mathrm{~mm})$, connected to a binary pump, and monitored using a photodiode array detector.

\section{Microbial strain}

The marine fungal isolate MR2012 used in this study was isolated from a Red Sea sediment in Hurghada, Egypt in September 2011, and taxonomically identified on a molecular basis as Aspergillus fumigatus ${ }^{21}$.

\section{Microbial fermentation, extraction, and isolation}

The fungal isolate MR2012 initially cultured on a solid medium composed of (g/L) glucose 10, yeast extract 10, malt extract 4. A 6 liter fermentation was conducted on a medium composed of $(\mathrm{g} / \mathrm{L})$ sucrose 100 , glucose 10 , casamino acids 0.1 , yeast extract 5, MOPS 21, $\mathrm{K}_{2} \mathrm{SO}_{4} 0.25 \times$ $10^{-6}, \mathrm{MgCl}_{2} .6 \mathrm{H}_{2} \mathrm{O} 1.0 \times 10^{-6}$ for 12 days at $30{ }^{\circ} \mathrm{C}$ with shaking at $180 \mathrm{rpm}$. At the end of the incubation period, Diaion HP-20 resin was added to the culture media and shaken for $6 \mathrm{~h}$ at 180 $\mathrm{rpm}$, then cultures were centrifuged (3000 rpm for $20 \mathrm{~min}$ ) where the residue composed of the fungal mycelia and resin were washed with distilled water twice and extracted with $\mathrm{MeOH}$, and subjected to LC-HRESIMS analysis. This extract was fractionated successively with $n$-hexane $(3 \times 250 \mathrm{~mL}), \mathrm{CH}_{2} \mathrm{Cl}_{2}(3 \times 300 \mathrm{~mL})$, and then EtOAc $(3 \times 250 \mathrm{~mL})$. Each solvent fraction was evaporated in vacuo and subjected to LC-HRESIMS and ${ }^{1} \mathrm{H}$ NMR analysis, which revealed that the $\mathrm{CH}_{2} \mathrm{Cl}_{2}$ fraction was the one of interest to follow. This $\mathrm{CH}_{2} \mathrm{Cl}_{2}$ fraction was loaded on Flash Biotage using a FLASH $65 \mathrm{i}$ cartridge, solvent $\mathrm{MeOH} /$ water $0-100 \%$, flow rate $60 \mathrm{~mL} / \mathrm{min}$ over 
$20 \mathrm{~min}$ and UV collection wavelengths 225 and $254 \mathrm{~nm}$ to produce 6 fractions. All of these fractions were monitored by LC-HRESIMS. Injection of fraction 4 into Agilent HPLC system using semi-preparative Sunfire $\mathrm{C}_{18}$ column $(250 \times 10 \mathrm{~mm}, 5 \mu \mathrm{m})$ with $\mathrm{CH}_{3} \mathrm{CN}: \mathrm{H}_{2} \mathrm{O} 30-90 \%$ over $30 \mathrm{~min}$ with a $2 \mathrm{~mL} / \mathrm{min}$ flow led to the isolation of $38 \mathrm{mg}$ of pseurotin $\mathrm{A}, 30 \mathrm{mg}$ of pseurotin $\mathrm{A}_{2}, 2 \mathrm{mg}$ of pseurotin $\mathrm{F} 1,6 \mathrm{mg}$ of 11-O-methylpseurotin A, and $5 \mathrm{mg}$ of pseurotin D. Injection of fraction 5 into Agilent HPLC system using semi-preparative Sunfire $\mathrm{C}_{18}$ column $(250 \times 10 \mathrm{~mm}, 5 \mu \mathrm{m})$ with $\mathrm{CH}_{3} \mathrm{CN}: \mathrm{H}_{2} \mathrm{O} 40-80 \%$ over $30 \mathrm{~min}$ with a $2 \mathrm{~mL} / \mathrm{min}$ flow led to the isolation of $26 \mathrm{mg}$ of azaspirofuran A and $23 \mathrm{mg}$ of azaspirofuran B.

\section{Compound preparation}

For experiments with zebrafish larvae, dry samples were dissolved in $100 \%$ dimethyl sulfoxide (DMSO, spectroscopy grade) as 100-fold concentrated stocks and diluted in embryo medium to a final concentration of $1 \%$ DMSO content. Control groups were treated with $1 \%$ DMSO (VHC) in accordance with the final solvent concentration of tested compounds. For mice experiments, a mixture of poly-ethylene glycol M.W. 200 (PEG200), 100 \% DMSO (spectroscopy grade), and demineralized water (PEG200:DMSO:water; 0.25:0.25:0.5) was used as solvent and VHC.

\section{Compound LogP prediction}

$\log \mathrm{P}(\mathrm{ACD} / \log \mathrm{P})$ values were obtained from ChemSpider and predicted by means of the ACD/Labs Percepta Platform (PhysChem Module) based on the compound structure ${ }^{50}$.

\section{Experimental animals}

All animal experiments carried out were approved by the Ethics Committee of the University of Leuven (approval numbers 101/2010, 061/2013, 150/2015, and 023/2017) and by the Belgian Federal Department of Public Health, Food Safety \& Environment (approval number LA1210199).

\section{Zebrafish}

Adult zebrafish (Danio rerio) stocks of AB strain (Zebrafish International Resource Center, Oregon, USA) were maintained at $28{ }^{\circ} \mathrm{C}$, on a 14/10-h light/dark cycle under standard 
aquaculture conditions. Fertilized eggs were collected via natural spawning and raised in embryo medium (1.5 mM HEPES, pH 7.2, $17.4 \mathrm{mM} \mathrm{NaCl}, 0.21 \mathrm{mM} \mathrm{KCl,} 0.12 \mathrm{mM} \mathrm{MgSO} 4$, and $0.18 \mathrm{mM} \mathrm{Ca}\left(\mathrm{NO}_{3}\right)_{2}$, and $0.6 \mu \mathrm{M}$ methylene blue) at $28^{\circ} \mathrm{C}$, under constant light.

Mice

Male NMRI mice (weight 18-20 g) were acquired from Charles River Laboratories and housed in poly-acrylic cages under a 14/10-h light/dark cycle at $21^{\circ} \mathrm{C}$. The animals were fed a pellet diet and water ad libitum, and were allowed to acclimate for one week before experimental procedures were conducted. Prior to the experiment, mice were isolated in a poly-acrylic cage with a pellet diet and water ad libitum for habituation overnight in the experimental room, to minimize stress.

\section{Zebrafish pentylenetetrazole seizure model}

\section{Toxicity evaluation}

Maximum tolerated concentration (MTC) was determined prior to further experiments and used as the highest test concentration. Experimental procedure was described before ${ }^{51}$. In brief, the MTC was investigated by exposing 12 larvae of $6 \mathrm{dpf}$ to a range of concentrations in a $100 \mu \mathrm{L}$ volume during $18 \mathrm{~h}$. The following parameters were investigated after 2 and $18 \mathrm{~h}$ of exposure: touch response, morphology, posture, edema, signs of necrosis, swim bladder, and heartbeat. MTC was defined as the highest concentration at which no larvae died nor showed signs of toxicity or locomotor impairment in comparison to VHC-treated control larvae. In case no MTC was reached, $100 \mu \mathrm{g} / \mathrm{mL}$ was used as the highest test concentration.

\section{Behavioral analysis}

Experimental procedure was described before ${ }^{17,20}$. In brief, a single 7-dpf larva (in case of $2 \mathrm{~h}$ incubation) or 6-dpf larva (in case of $18 \mathrm{~h}$ incubation) was placed in each well of a 96-well plate and treated with either VHC (1\% DMSO) or compound in a $100 \mu \mathrm{L}$ volume. Larvae were incubated in dark for 2 or $18 \mathrm{~h}$ at $28^{\circ} \mathrm{C}$, whereafter $100 \mu \mathrm{L}$ of either VHC (embryo medium) or $40 \mathrm{mM}$ PTZ was added to each well. Next, within 5 min the 96-well plate was placed in an automated tracking device (ZebraBox Viewpoint, France) and larval behavior was video recorded for $30 \mathrm{~min}$. The complete procedure was performed in dark conditions using infrared light. Total locomotor activity was recorded by ZebraLab software (Viewpoint, France) and 
expressed in actinteg units, which is the sum of pixel changes detected during the defined time interval (5 min). Larval behavior was depicted as mean actinteg values in the 30-min recording period and over 5 min time intervals. Data are expressed as mean \pm SD or as mean \pm SEM when the means of independent experiments were pooled.

\section{Electrophysiology}

Experimental procedure was described before ${ }^{51-53}$. In brief, non-invasive LFP recordings were measured from the midbrain of 7-dpf zebrafish larvae pre-incubated with VHC only, PTZ only, compound and VHC, or compound and PTZ. Larvae were incubated for approximately 2 or 18 $\mathrm{h}$ with VHC (1 \% DMSO) or compound in a $100 \mu \mathrm{L}$ volume $\left(28^{\circ} \mathrm{C}\right)$. After incubation, $100 \mu \mathrm{L}$ VHC (embryo medium) or $40 \mathrm{mM}$ PTZ (20 mM working concentration) was added to the well for $15 \mathrm{~min}\left(28{ }^{\circ} \mathrm{C}\right)$ prior to the recording. For electrophysiological recordings (room temperature), larvae were immobilized in $2 \%$ low melting point agarose (Invitrogen) and the signal electrode [an electrode inside a soda-glass pipet (1412227, Hilgenberg) pulled with a DMZ Universal Puller (Zeitz, Germany), diameter \pm 20 microns, containing artificial cerebrospinal fluid (ACSF: $124 \mathrm{mM} \mathrm{NaCl}, 10 \mathrm{mM}$ glucose, $2 \mathrm{mM} \mathrm{KCl}, 2 \mathrm{mM} \mathrm{MgSO} 4,2 \mathrm{mM}$ $\mathrm{CaCl}_{2}, 1.25 \mathrm{mM} \mathrm{KH}_{2} \mathrm{PO}_{4}$, and $\left.\left.26 \mathrm{mM} \mathrm{NaHCO}_{3}, 300-310 \mathrm{mOsmols}\right)\right]$ was positioned on the skin covering the optic tectum. Each recording lasted $600 \mathrm{~s}$ and was analyzed manually by quantifying the number, cumulative duration, and mean duration of epileptiform-like events with Clampfit 10.2 software (Molecular Devices Corporation, USA). An electrical discharge was considered epileptiform if it was a polyspiking event comprising at least 3 spikes with a minimum amplitude of three times the baseline amplitude and a duration of at least $100 \mathrm{~ms}$. Data are expressed as mean \pm SD.

\section{Mouse 6-Hz (44 mA) psychomotor seizure model}

Experimental procedure was described before ${ }^{19,45,51}$. In brief, NMRI mice (average weight 28 $\mathrm{g}$, range 23-32 g) were randomly divided into control and treatment groups $(\mathrm{n}=5-10) .50 \mu \mathrm{L}$ (injection volume was adjusted to the individual weight) of VHC (PEG200:DMSO:water; 0.25:0.25:0.5) or treatment (an ASD or test compound dissolved in VHC) was i.p. injected in mice and after 30 or 120 min (in case of phenytoin, as reported by Barton and colleagues ${ }^{41}$ ) psychomotor seizures were induced by low frequency, long duration corneal electrical stimulation (6 Hz, $0.2 \mathrm{~ms}$, rectangular pulse width, $3 \mathrm{~s}$ duration, $44 \mathrm{~mA}$ ) using an ECT Unit 5780 (Ugo Basile, Comerio, Italy). Mice were manually restrained and a drop of ocular 
anesthetic ( $0.5 \%$ lidocaine) was applied to the corneas before stimulation. Following electrical current stimulation, the mouse was released in a transparent cage for behavioral observation, which was video-recorded. VHC-treated mice typically displayed stun, twitching of the vibrissae, forelimb clonus, and Straub tail. In addition, facial and mouth jerking as well as head nodding were observed occasionally. Seizure durations were measured during the experiment by experienced researchers, familiar with the different seizure behaviors. In addition, seizure durations were determined by blinded video analysis to confirm or correct the initial observations. Data are expressed as mean \pm SD.

\section{ADMET profiling}

\section{Cell viability and MTT assays}

Experimental procedure was described before ${ }^{54}$. Three cell lines were used: a) Hep G2 (HB8065), a well-differentiated human hepatocellular carcinoma cell line, b) THLE-2, human liver epithelial cells transformed with SV40 large T-antigen and c) SHSY5Y, a thrice-cloned subline of a human metastatic bone tumor. Cells were seeded at a concentration of $1 \times 10^{4}$ cells/well in $200 \mu \mathrm{L}$ culture medium and incubated at $37^{\circ} \mathrm{C}$ in $5 \% \mathrm{CO}_{2}$ using 96-well plates for $24 \mathrm{~h}$. Next, the medium was replaced with medium complemented with test compounds at different concentrations. After another $24 \mathrm{~h}$ incubation, the medium was replaced by $100 \mu \mathrm{L}$ of a MTT (3-(4,5-dimethylthiazol-2-yl)-2,5-diphenyltetrazolium bromide) solution (5 mg/mL in PBS and diluted at $0.5 \mathrm{mg} / \mathrm{mL}$ in MEM without phenol red). The plates were gently shaken and incubated for $3 \mathrm{~h}$ at $37^{\circ} \mathrm{C}$ in a $5 \% \mathrm{CO}_{2}$ incubator. The supernatant was removed and $100 \mu \mathrm{L}$ of $100 \%$ DMSO was added. The plates were gently shaken to solubilize the formed formazan. The absorbance was measured using a multireader (Victor2 (Wallac)) at a wavelength of $570 \mathrm{~nm}$.

\section{Cardiotoxicity}

Fluorescence-based assays were performed using HEK293 cell lines that stably express the Nav1.5-channel, Cav1.2-channel, or hERG $\mathrm{K}^{+}$-channel, using FMP Red Dye (Molecular Devices), calcium-sensitive fluorescent dye Fluo-4 (Invitrogen) and FluxOR ${ }^{\mathrm{TM}}$ reagent (Invitrogen), respectively, and a FLIPR Tetra High-Throughput Cellular Screening System (Molecular Devices), according to manufacturer's protocols (Molecular Devices). Tetrodotoxin, israpidine and nicarpidine, and astemizole and haloperidol were used as standard 
sodium channel, calcium channel, and hERG channel blockers. Data were analyzed using Genedata Screener.

\section{CYP450 enzyme inhibition assay}

Experimental procedure was described before ${ }^{55}$. Assessment of CYP450 inhibition was conducted in 96-well plate format at $37{ }^{\circ} \mathrm{C}$. Test compounds were dissolved in DMSO/acetonitrile (ACN) $(2 \mu \mathrm{L})$ and diluted in $98 \mu \mathrm{L}$ NADPH solution $(2 \mathrm{mM})$. Reactions were started by addition of $100 \mu \mathrm{L}$ potassium phosphate buffer $(200 \mathrm{mM}, \mathrm{pH} 7.4)$ containing Human Liver Microsomes (HLM) $(0.5 \mathrm{mg} / \mathrm{mL})$. Probe reactions for CYP3A4, CYP2D6 and CYP2C9 were conducted with $50 \mu \mathrm{M}$ testosterone, $22 \mu \mathrm{M}$ dextromethorphan and $10 \mu \mathrm{M}$ diclofenac for $15 \mathrm{~min}$. Reactions were terminated with the addition of a quench solution (90 $\mu \mathrm{L}$ ) of ACN containing internal standards for LC-MS/MS determination (60 ppb cortisone, 100 ppb 4'-hydroxydiclofenac- ${ }^{13} \mathrm{C}_{6}, 60 \mathrm{ppb}$ levallorphan).

\section{Metabolic stability assay}

The assay was performed with a mixture of test compounds $(1 \mu \mathrm{M}), \mathrm{NADPH}(4 \mathrm{mM})$ and HLM $(1 \mathrm{mg} / \mathrm{mL})$ incubated at $37^{\circ} \mathrm{C}$. Reactions were quenched at $0,15,30,45,60$, and $90 \mathrm{~min}$, using an equal volume of ACN and then diluted 1:1 with water prior to analysis by LC-MS/MS.

The analysis was performed using an Agilent Series 1290 LC system (Agilent Technologies, Santa Clara, CA, USA) using a Supelco Discovery HS C $18(2.1 \times 50 \mathrm{~mm}) 3 \mu \mathrm{m}$ column that was held at $30{ }^{\circ} \mathrm{C}$. Solvent A contained water with $0.1 \%$ formic acid and solvent B contained ACN with $0.1 \%$ formic acid, and the flow rate was set at $400 \mu \mathrm{L} / \mathrm{min}$. The gradient elution was performed as follows: $0-0.5 \min 0 \%$ eluent $\mathrm{B}$; $0.5-7$ min $100 \%$ eluent $\mathrm{B} ; 7-9 \min 100 \%$ eluent B; 9-9.2 min $0 \%$ eluent B; and 9.2-10.5 min $0 \%$ eluent B. An API 4000 mass spectrometer in positive ESI mode (AB SCIEX, Concord, ON, Canada) was used with a generic method for data acquisition on all compounds. Data processing was performed using MultiQuant Software (AB SCIEX, Concord, ON, Canada) to process the data. Peak areas were used to plot the Ln \% remaining relative to time $(t)=0$. The slope of the natural $\log$ of the percent remaining versus time was calculated to determine the first-order rate constant $(k)$ and the half-life $\left(t_{1 / 2}\right)$ of the test compounds according to the following equation: $\mathrm{t}_{1 / 2}=0.693 / \mathrm{k}(\mathrm{min})$ 


\section{Kinetic solubility assay}

Experimental procedure was described before ${ }^{56}$. The kinetic solubility assay was conducted in 96-well, flat-bottom, transparent polystyrene plates (Costar 9018, Corning, Tewksbury MA). Six two-fold serial dilutions of an initial $10 \mathrm{mM}$ test compound solution were prepared in DMSO. After a 2-h incubation period (to avoid missing slow precipitation) absorbance was measured at $620 \mathrm{~nm}$ by an EnVision multilabel plate reader. The kinetic solubility was estimated from the concentration of test compound that produced an increase in absorbance above the background levels (i.e., $1 \%$ DMSO in buffer).

\section{Plasma protein binding assay}

Experimental procedure was described before ${ }^{57}$. Rapid equilibrium dialysis was performed with RED device inserts (Thermo Scientific, Meridian Rd., Rockford, IL) containing dialysis membrane with a molecular weight cut-off of 8000 Daltons. Serum $(200 \mu L)$ containing test compound $(5 \mu \mathrm{M})$ was added to the serum chamber of the insert and $350 \mu \mathrm{L}$ of buffer was added to the buffer chamber of the insert. Dialysis was done at $37^{\circ} \mathrm{C}$ with shaking at $100 \mathrm{rpm}$ for $5 \mathrm{~h}$. Following dialysis, an aliquot of $50 \mu \mathrm{L}$ was removed from each well (plasma and buffer side) and diluted with an equal volume of opposite matrix to nullify the matrix effect. Then a fraction $(50 \mu \mathrm{L})$ of each dialyzed sample was crashed with $150 \mu \mathrm{L}$ of ACN containing internal standard and vortexed for $1 \mathrm{~min}$. The samples were centrifuged at $13,300 \mathrm{rpm}$ at $4{ }^{\circ} \mathrm{C}$ for 12 min and $100 \mu \mathrm{L}$ of supernatant was used for LC-MS/MS analysis.

\section{Parallel artificial membrane permeability assay (PAMPA)}

The Gentest Pre-coated PAMPA Plate System (Corning) was used to perform the permeability assays. The 96-well filter plate, pre-coated with lipids, was used as the permeation acceptor and a matching 96-well receiver plate was used as the permeation donor. Compound solutions were prepared by diluting $10 \mathrm{mM}$ DMSO stock solutions in PBS with a final concentration of 10 $\mu \mathrm{M}$. The compound solutions were added to the wells (300 $\mu \mathrm{L} /$ well) of the receiver plate and PBS was added to the wells $(200 \mu \mathrm{L} /$ well $)$ of the pre-coated filter plate. The filter plate was then coupled with the receiver plate and the plate assembly was incubated at room temperature without agitation for $5 \mathrm{~h}$. Next, the plates were separated and $50 \mu \mathrm{L}$ solution from each well of both the filter plate and the receiver plate was transferred to a vial with $150 \mu \mathrm{L}$ ACN and centrifuged at $13,300 \mathrm{rpm}$ for $10 \mathrm{~min}$ at $4{ }^{\circ} \mathrm{C}$. The supernatant was diluted in a solution 
water/ACN (50/50). The final concentration of compounds in both donor wells and acceptor wells was analyzed by LC-MS/MS. Permeability of the compounds was calculated using the following equation:

Permeability $(\mathrm{cm} / \mathrm{s}): \mathrm{Pe}=\{-\ln [1-\mathrm{CA}(\mathrm{t}) / \mathrm{Ceq}]\} /[\mathrm{A} *(1 / \mathrm{VD}+1 / \mathrm{VA}) * \mathrm{t}]\}$

$\mathrm{A}=$ filter area $\left(0.3 \mathrm{~cm}^{2}\right), \mathrm{VD}=$ donor well volume $(0.3 \mathrm{~mL}), \mathrm{VA}=$ acceptor well volume $(0.2$ $\mathrm{mL}), \mathrm{t}=$ incubation time $(\mathrm{s}), \mathrm{CA}(\mathrm{t})=$ compound concentration in acceptor well at time $\mathrm{t}, \mathrm{CD}(\mathrm{t})$ $=$ compound concentration in donor well at time $t$, and Ceq = $(\mathrm{CD}(\mathrm{t}) * \mathrm{VD}+\mathrm{CA}(\mathrm{t}) * \mathrm{VA}) /(\mathrm{VD}+\mathrm{VA})$

\section{ABBREVIATIONS}

ACN, acetonitrile; ACSF, artificial cerebrospinal fluid; ASD, antiseizure drug; dpf, days postfertilization; DMSO, dimethyl sulfoxide; FP7, Seventh Framework Programme; h, hours; HLM, Human Liver Microsomes; LFP, local field potential; min, minute; MTC, maximum tolerated concentration; PEG200, poly-ethylene glycol M.W. 200; PTZ, pentylenetetrazole; s, seconds; t1/2, half-life; VHC, vehicle

\section{AUTHOR INFORMATION}

\section{*Corresponding authors}

Prof. Dr. Peter A. M. de Witte, Laboratory for Molecular Biodiscovery, Department of Pharmaceutical and Pharmacological Sciences, KU Leuven, Herestraat 49 box 824, 3000 Leuven, Belgium. E-mail: peter.dewitte@kuleuven.be

Prof. Dr. Marcel Jaspars, Marine Biodiscovery Centre, Department of Chemistry, University of Aberdeen, Aberdeen AB24 3UE, Scotland, UK. E-mail: m.jaspars@abdn.ac.uk

\section{Author contributions}

PAMW, MJ, DC, and MR were responsible for study design. PAMW, MJ, DC, MR, FR, and RE were responsible for experimental design. Experiments were performed by DC, MR, JNT, MPB, ND, RV, CD, JP del P, and AJS. DC and MR were responsible for data acquisition and 
analysis. DC, MR, and PAMW wrote the manuscript. DC prepared the figures and tables. All authors edited and approved the final version of the manuscript.

\section{Funding sources}

This work was supported by the PharmaSea project (www.pharma-sea.eu), funded by the EU Seventh Framework Programme (contract number 312184).

\section{Conflict of interest}

There is no potential conflict of interest.

\section{ACKNOWLEDGEMENT}

This work was supported by the PharmaSea project (www.pharma-sea.eu), funded by the EU Seventh Framework Programme (contract number 312184). We would like to acknowledge Dr. Francisca Vicente, Dr. Carmen Ramos, and Dr. Bastien Cautain for their contribution with regards to the ADMET analysis. Concerning the graphical table of contents, we acknowledge somersault18:24 BVBA (www.somersault1824.com) for providing scientific illustrations.

\section{SUPPORTING INFORMATION}

1D NMR data of pseurotin A, pseurotin $\mathrm{A}_{2}$, pseurotin F1, 11-O-methylpseurotin A, pseurotin $\mathrm{D}$, and azaspirofurans $\mathrm{A}$ and $\mathrm{B}$.

\section{REFERENCES}

[1] Ngugi, A. K., Bottomley, C., Kleinschmidt, I., Sander, J. W., and Newton, C. R. (2010) Estimation of the burden of active and life-time epilepsy: a meta-analytic approach, Epilepsia 51, 883-890.

[2] Sander, J. W. (2003) The epidemiology of epilepsy revisited, Curr Opin Neurol 16, 165170.

[3] Singh, A., and Trevick, S. (2016) The Epidemiology of Global Epilepsy, Neurol Clin 34, 837-847.

[4] Fisher, R. S. (2015) Redefining epilepsy, Curr Opin Neurol 28, 130-135. 
[5] Franco, V., French, J. A., and Perucca, E. (2016) Challenges in the clinical development of new antiepileptic drugs, Pharmacol Res 103, 95-104.

[6] Golyala, A., and Kwan, P. (2017) Drug development for refractory epilepsy: The past 25 years and beyond, Seizure 44, 147-156.

[7] Bialer, M., Johannessen, S. I., Levy, R. H., Perucca, E., Tomson, T., and White, H. S. (2017) Progress report on new antiepileptic drugs: A summary of the Thirteenth Eilat Conference on New Antiepileptic Drugs and Devices (EILAT XIII), Epilepsia 58, 181221.

[8] Jaspars, M., De Pascale, D., Andersen, J. H., Reyes, F., Crawford, A. D., and Ianora, A. (2016) The marine biodiscovery pipeline and ocean medicines of tomorrow, Journal of the Marine Biological Association of the United Kingdom 96, 151-158.

[9] Kong, D. X., Jiang, Y. Y., and Zhang, H. Y. (2010) Marine natural products as sources of novel scaffolds: achievement and concern, Drug Discov Today 15, 884-886.

[10] Sakai, R., and Swanson, G. T. (2014) Recent progress in neuroactive marine natural products, Nat Prod Rep 31, 273-309.

[11] West, K. H., and Crawford, A. D. (2016) Marine Biodiscovery Goes Deeper: Using In Vivo Bioassays Based on Model Organisms to Identify Biomedically Relevant Marine Metabolites, Planta Med 82, 754-760.

[12] Howe, K., and Clark, M. D., and Torroja, C. F., and Torrance, J., and Berthelot, C., and Muffato, M., and Collins, J. E., and Humphray, S., and McLaren, K., and Matthews, L., and McLaren, S., and Sealy, I., and Caccamo, M., and Churcher, C., and Scott, C., and Barrett, J. C., and Koch, R., and Rauch, G. J., and White, S., and Chow, W., and Kilian, B., and Quintais, L. T., and Guerra-Assuncao, J. A., and Zhou, Y., and Gu, Y., and Yen, J., and Vogel, J. H., and Eyre, T., and Redmond, S., and Banerjee, R., and Chi, J., and Fu, B., and Langley, E., and Maguire, S. F., and Laird, G. K., and Lloyd, D., and Kenyon, E., and Donaldson, S., and Sehra, H., and Almeida-King, J., and Loveland, J., and Trevanion, S., and Jones, M., and Quail, M., and Willey, D., and Hunt, A., and Burton, J., and Sims, S., and McLay, K., and Plumb, B., and Davis, J., and Clee, C., and Oliver, K., and Clark, R., and Riddle, C., and Elliot, D., and Threadgold, G., and Harden, G., and Ware, D., and Begum, S., and Mortimore, B., and Kerry, G., and Heath, P., and Phillimore, B., and Tracey, A., and Corby, N., and Dunn, M., and Johnson, C., and Wood, J., and Clark, S., and Pelan, S., and Griffiths, G., and Smith, M., and Glithero, R., and Howden, P., and Barker, N., and Lloyd, C., and Stevens, C., and Harley, J., and Holt, K., and Panagiotidis, G., and Lovell, J., and Beasley, H., and Henderson, C., and Gordon, D., and Auger, K., and Wright, D., and Collins, J., and Raisen, C., and Dyer, L., and Leung, K., and Robertson, L., and Ambridge, K., and Leongamornlert, D., and McGuire, S., and Gilderthorp, R., and Griffiths, C., and Manthravadi, D., and Nichol, S., and Barker, G., and Whitehead, S., and Kay, M., and Brown, J., and Murnane, C., and Gray, E., and Humphries, M., and Sycamore, N., and Barker, D., and Saunders, D., and Wallis, J., and Babbage, A., and Hammond, S., and Mashreghi-Mohammadi, M., and Barr, L., and Martin, S., and Wray, P., and Ellington, A., and Matthews, N., and Ellwood, M., and Woodmansey, R., and Clark, G., and Cooper, J., and Tromans, A., and Grafham, D., and Skuce, C., and Pandian, R., and Andrews, R., and Harrison, E., and Kimberley, A., and Garnett, J., and Fosker, N., and Hall, R., and Garner, P., and Kelly, D., and Bird, C., and Palmer, S., and Gehring, I., and Berger, A., and Dooley, C. M., and Ersan-Urun, Z., and Eser, C., and Geiger, H., and Geisler, M., and Karotki, L., and Kirn, A., and Konantz, J., and Konantz, M., and Oberlander, M., and RudolphGeiger, S., and Teucke, M., and Lanz, C., and Raddatz, G., and Osoegawa, K., and Zhu, B., and Rapp, A., and Widaa, S., and Langford, C., and Yang, F., and Schuster, S. C., and Carter, N. P., and Harrow, J., and Ning, Z., and Herrero, J., and Searle, S. M., and 
Enright, A., and Geisler, R., and Plasterk, R. H., and Lee, C., and Westerfield, M., and de Jong, P. J., and Zon, L. I., and Postlethwait, J. H., and Nusslein-Volhard, C., and Hubbard, T. J., and Roest Crollius, H., and Rogers, J., and Stemple, D. L. (2013) The zebrafish reference genome sequence and its relationship to the human genome, Nature 496, 498-503.

[13] MacRae, C. A., and Peterson, R. T. (2015) Zebrafish as tools for drug discovery, Nat Rev Drug Discov 14, 721-731.

[14] Khan, K. M., Collier, A. D., Meshalkina, D. A., Kysil, E. V., Khatsko, S. L., Kolesnikova, T., Morzherin, Y. Y., Warnick, J. E., Kalueff, A. V., and Echevarria, D. J. (2017) Zebrafish models in neuropsychopharmacology and CNS drug discovery, $\mathrm{Br} J$ Pharmacol.

[15] Copmans, D., Siekierska, A., and de Witte, P. A. M. (2017) Zebrafish Models of Epilepsy and Epileptic Seizures, In Models of Seizures and Epilepsy (Pitkänen, A., Buckmaster, P. S., Galanopoulou, A. S., and Moshé, S. L., Eds.) Second ed., pp 369-384, Elsevier.

[16] Baraban, S. C., Taylor, M. R., Castro, P. A., and Baier, H. (2005) Pentylenetetrazole induced changes in zebrafish behavior, neural activity and c-fos expression, Neuroscience 131, 759-768.

[17] Afrikanova, T., Serruys, A. S., Buenafe, O. E., Clinckers, R., Smolders, I., de Witte, P. A., Crawford, A. D., and Esguerra, C. V. (2013) Validation of the zebrafish pentylenetetrazol seizure model: locomotor versus electrographic responses to antiepileptic drugs, PLoS One 8, e54166.

[18] Berghmans, S., Hunt, J., Roach, A., and Goldsmith, P. (2007) Zebrafish offer the potential for a primary screen to identify a wide variety of potential anticonvulsants, Epilepsy Res $75,18-28$.

[19] Buenafe, O. E., Orellana-Paucar, A., Maes, J., Huang, H., Ying, X., De Borggraeve, W., Crawford, A. D., Luyten, W., Esguerra, C. V., and de Witte, P. (2013) Tanshinone IIA exhibits anticonvulsant activity in zebrafish and mouse seizure models, ACS Chem Neurosci 4, 1479-1487.

[20] Orellana-Paucar, A. M., Serruys, A. S., Afrikanova, T., Maes, J., De Borggraeve, W., Alen, J., Leon-Tamariz, F., Wilches-Arizabala, I. M., Crawford, A. D., de Witte, P. A., and Esguerra, C. V. (2012) Anticonvulsant activity of bisabolene sesquiterpenoids of Curcuma longa in zebrafish and mouse seizure models, Epilepsy Behav 24, 14-22.

[21] El-Gendy, B. E.-D. M., and Rateb, M. E. (2015) Antibacterial activity of diketopiperazines isolated from a marine fungus using t-butoxycarbonyl group as a simple tool for purification, Bioorganic \& Medicinal Chemistry Letters 25, 3125-3128.

[22] Komagata, D., Fujita, S., Yamashita, N., Saito, S., and Morino, T. (1996) Novel neuritogenic activities of pseurotin A and penicillic acid, J Antibiot (Tokyo) 49, 958959.

[23] Wink, J. D., Grabley, S. D., Gareis, M. D., Zeeck, A. P. D., and Philipps, S. D. (1993) Biologically active pseurotin A and D, new metabolites from Aspergillus fumigatus, process for their preparation and their use as apomorphine antagonists, In Espacenet.

[24] Mehedi, M. A. U., Molla, A. H., Khondkar, P., Sultana, S., Islam, M. A., Rashid, M. A., and Chowdhury, R. (2010) Pseurotin A: An Antibacterial Secondary Metabolite from Aspergillus fumigatus, Asian Journal of Chemistry 22, 2611-2614.

[25] Martinez-Luis, S., Cherigo, L., Arnold, E., Spadafora, C., Gerwick, W. H., and CubillaRios, L. (2012) Antiparasitic and anticancer constituents of the endophytic fungus Aspergillus sp. strain F1544, Nat Prod Commun 7, 165-168.

[26] Ishikawa, M., Ninomiya, T., Akabane, H., Kushida, N., Tsujiuchi, G., Ohyama, M., Gomi, S., Shito, K., and Murata, T. (2009) Pseurotin A and its analogues as inhibitors of immunoglobuline E production, Bioorg Med Chem Lett 19, 1457-1460. 
[27] Wang, F.-Z., Li, D.-H., Zhu, T.-J., Zhang, M., and Gu, Q.-Q. (2011) Pseurotin A1 and A2, two new 1-oxa-7-azaspiro[4.4]non-2-ene-4,6-diones from the holothurian-derived fungus Aspergillus fumigatus WFZ-25, Canadian Journal of Chemistry 89, 72-76.

[28] Saraiva, N. N., Rodrigues, B. S., Jimenez, P. C., Guimaraes, L. A., Torres, M. C., Rodrigues-Filho, E., Pfenning, L. H., Abreu, L. M., Mafezoli, J., de Mattos, M. C., Costa-Lotufo, L. V., and de Oliveira Mda, C. (2015) Cytotoxic compounds from the marine-derived fungus Aspergillus sp. recovered from the sediments of the Brazilian coast, Nat Prod Res 29, 1545-1550.

[29] Lee, M. S., Wang, S. W., Wang, G. J., Pang, K. L., Lee, C. K., Kuo, Y. H., Cha, H. J., Lin, R. K., and Lee, T. H. (2016) Angiogenesis Inhibitors and Anti-Inflammatory Agents from Phoma sp. NTOU4195, J Nat Prod 79, 2983-2990.

[30] Wenke, J., Anke, H., and Sterner, O. (1993) Pseurotin A and 8-O-Demethylpseurotin A from Aspergillus fumigatus and Their Inhibitory Activities on Chitin Synthase, Bioscience, Biotechnology, and Biochemistry 57, 961-964.

[31] Shi, Y. S., Zhang, Y., Chen, X. Z., Zhang, N., and Liu, Y. B. (2015) Metabolites Produced by the Endophytic Fungus Aspergillus fumigatus from the Stem of Erythrophloeum fordii Oliv, Molecules 20, 10793-10799.

[32] Ren, H., Liu, R., Chen, L., Zhu, T., Zhu, W. M., and Gu, Q. Q. (2010) Two new heterospirocyclic $\gamma$-lactam derivatives from marine sediment-derived fungus Aspergillus sydowi D2-6, Archives of Pharmacal Research 33, 499-502.

[33] Ren, H., Cao, X.-L., Wang, Q.-E., and Xv, C.-M. (2011) Antitumor metabolites from fungus Aspergillus sydowi D 2-6, Chinese Pharmaceutical Journal 46, 569-575.

[34] Rateb, M. E., Hallyburton, I., Houssen, W. E., Bull, A. T., Goodfellow, M., Santhanam, R., Jaspars, M., and Ebel, R. (2013) Induction of diverse secondary metabolites in Aspergillus fumigatus by microbial co-culture, RSC Advances 3, 14444-14450.

[35] Wink, J., Grabley, S., Gareis, M., Thiericke, R., and Kirsch, R. (1993) Pseurotin F1/F2, new metabolites from Aspergillus fumigatus, process for their preparation and their use as apomorphine antagonists In Europe PMC.

[36] Breitenstein, W., Chexal, K. K., Mohr, P., and Tamm, C. (1981) Pseurotin B, C, D, and E. Further New Metabolites of Pseudeurotium ovalis STOLK, Helvetica Chimica Acta 64, 379-388.

[37] Wakefield, J., Hassan, H. M., Jaspars, M., Ebel, R., and Rateb, M. E. (2017) Dual Induction of New Microbial Secondary Metabolites by Fungal Bacterial Co-cultivation, Front Microbiol 8, 1284.

[38] Milan, D. J., Peterson, T. A., Ruskin, J. N., Peterson, R. T., and MacRae, C. A. (2003) Drugs that induce repolarization abnormalities cause bradycardia in zebrafish, Circulation 107, 1355-1358.

[39] Britton, J. W., Frey, L. C., Hopp, J. L., Korb, P., Koubeissi, M. Z., Lievens, W. E., PestanaKnight, E. M., and St. Louis, E. K. (2016) In Electroencephalography (EEG): An Introductory Text and Atlas of Normal and Abnormal Findings in Adults, Children, and Infants (St. Louis, E. K., and Frey, L. C., Eds.), Chicago.

[40] Zdebik, A. A., Mahmood, F., Stanescu, H. C., Kleta, R., Bockenhauer, D., and Russell, C. (2013) Epilepsy in kcnj10 morphant zebrafish assessed with a novel method for longterm EEG recordings, PLoS One 8, e79765.

[41] Barton, M. E., Klein, B. D., Wolf, H. H., and White, H. S. (2001) Pharmacological characterization of the $6 \mathrm{~Hz}$ psychomotor seizure model of partial epilepsy, Epilepsy Res 47, 217-227.

[42] Wilcox, K. S., Dixon-Salazar, T., Sills, G. J., Ben-Menachem, E., White, H. S., Porter, R. J., Dichter, M. A., Moshe, S. L., Noebels, J. L., Privitera, M. D., and Rogawski, M. A. 
(2013) Issues related to development of new antiseizure treatments, Epilepsia 54 Suppl 4, 24-34.

[43] Fisher, R. S., Cross, J. H., D'Souza, C., French, J. A., Haut, S. R., Higurashi, N., Hirsch, E., Jansen, F. E., Lagae, L., Moshe, S. L., Peltola, J., Roulet Perez, E., Scheffer, I. E., Schulze-Bonhage, A., Somerville, E., Sperling, M., Yacubian, E. M., and Zuberi, S. M. (2017) Instruction manual for the ILAE 2017 operational classification of seizure types, Epilepsia 58, 531-542.

[44] Holcomb, M. J., and Dean, R. S. (2011) Psychomotor Seizures, In Encyclopedia of Child Behavior and Development (Goldstein, S., and Naglieri, J. A., Eds.), pp 1191-1192, Springer US, Boston, MA.

[45] Orellana-Paucar, A. M., Afrikanova, T., Thomas, J., Aibuldinov, Y. K., Dehaen, W., de Witte, P. A., and Esguerra, C. V. (2013) Insights from zebrafish and mouse models on the activity and safety of ar-turmerone as a potential drug candidate for the treatment of epilepsy, PLoS One 8, e81634.

[46] Walrave, L., Maes, K., Coppens, J., Bentea, E., Van Eeckhaut, A., Massie, A., Van Liefferinge, J., and Smolders, I. (2015) Validation of the $6 \mathrm{~Hz}$ refractory seizure mouse model for intracerebroventricularly administered compounds, Epilepsy Res 115, 67-72.

[47] Leclercq, K., Afrikanova, T., Langlois, M., De Prins, A., Buenafe, O. E., Rospo, C. C., Van Eeckhaut, A., de Witte, P. A., Crawford, A. D., Smolders, I., Esguerra, C. V., and Kaminski, R. M. (2015) Cross-species pharmacological characterization of the allylglycine seizure model in mice and larval zebrafish, Epilepsy Behav 45, 53-63.

[48] Dey, A., Kang, X., Qiu, J., Du, Y., and Jiang, J. (2016) Anti-Inflammatory Small Molecules To Treat Seizures and Epilepsy: From Bench to Bedside, Trends Pharmacol Sci 37, 463-484.

[49] Vezzani, A. (2015) Anti-inflammatory drugs in epilepsy: does it impact epileptogenesis?, Expert Opin Drug Saf 14, 583-592.

[50] Petrauskas, A. A., and Kolovanov, E. A. (2000) ACD/Log P method description, Perspectives in Drug Discovery and Design 19, 99-116.

[51] Copmans, D., Orellana-Paucar, A. M., Steurs, G., Zhang, Y., Ny, A., Foubert, K., Exarchou, V., Siekierska, A., Kim, Y., De Borggraeve, W., Dehaen, W., Pieters, L., and de Witte, P. A. M. (2018) Methylated flavonoids as anti-seizure agents: Naringenin 4',7dimethyl ether attenuates epileptic seizures in zebrafish and mouse models, Neurochem Int 112, 124-133.

[52] Sourbron, J., Smolders, I., de Witte, P., and Lagae, L. (2017) Pharmacological Analysis of the Anti-epileptic Mechanisms of Fenfluramine in scn1a Mutant Zebrafish, Frontiers in Pharmacology 8.

[53] Zhang, Y., Vanmeert, M., Siekierska, A., Ny, A., John, J., Callewaert, G., Lescrinier, E., Dehaen, W., de Witte, P. A. M., and Kaminski, R. M. (2017) Inhibition of glutamate decarboxylase (GAD) by ethyl ketopentenoate (EKP) induces treatment-resistant epileptic seizures in zebrafish, Sci Rep 7, 7195.

[54] Patel, R. M., and Patel, S. K. (2011) Cytotoxic activity of methanolic extract of Artocarpus heterophyllus against A549, Hela and MCF-7 cell lines., Journal of Applied Pharmaceutical Science 1, 167-171.

[55] Perez-Del Palacio, J., Diaz, C., Vergara, N., Algieri, F., Rodriguez-Nogales, A., de Pedro, N., Rodriguez-Cabezas, M. E., Genilloud, O., Galvez, J., and Vicente, F. (2017) Exploring the Role of CYP3A4 Mediated Drug Metabolism in the Pharmacological Modulation of Nitric Oxide Production, Front Pharmacol 8, 202.

[56] Perez, J., Diaz, C., Asensio, F., Palafox, A., Genilloud, O., and Vicente, F. (2015) A novel in vitro approach for simultaneous evaluation of CYP3A4 inhibition and kinetic aqueous solubility, J Biomol Screen 20, 254-264. 
[57] Kumar Singh, J., Solanki, A., Maniyar, R. C., Banerjee, D., and Shirsath, V. S. (2012) Rapid Equilibrium Dialysis (RED): An In-Vitro High-Throughput Screening Technique for Plasma Protein Binding Using Human and Rat Plasma, Journal of Bioequivalence \& Bioavailability. 


\section{FIGURE LEGENDS}

Figure 1. Chemical structures isolated from extracts of the marine fungus Aspergillus fumigatus.

Figure 2. Behavioral antiseizure analysis in the zebrafish PTZ seizure model. Antiseizure activity analysis of compounds at their maximum tolerated concentrations (MTC, Table 1) in the zebrafish pentylenetetrazole (PTZ) seizure model after $2 \mathrm{~h}(\mathrm{~A})$ and $18 \mathrm{~h}$ (B) of incubation. PTZ-induced seizure-like behavior is expressed as mean actinteg units $/ 5 \min ( \pm \mathrm{SD})$ during the 30-min recording period. Number of replicate wells per condition: $n=48$ (A) and $n=70-71$ (B) for VHC + PTZ and VHC + VHC controls, and n = 8-12 (A) and n =9-14 (B) for compound + PTZ conditions. Data are pooled from multiple experimental plates. Statistical analysis: oneway ANOVA with Dunnett's multiple comparison test (GraphPad Prism 5). Significance levels: $* \mathrm{p} \leq 0.05 ; * * \mathrm{p} \leq 0.01 ; * * * \mathrm{p} \leq 0.001$.

Figure 3. Behavioral antiseizure analysis of azaspirofuran $A$ and pseurotin $A_{2}$ in the zebrafish PTZ seizure model. Antiseizure activity of azaspirofuran A (A-B) and pseurotin A2 (C-D) in the zebrafish pentylenetetrazole (PTZ) seizure model after $2 \mathrm{~h}$ (A-B) and $18 \mathrm{~h}(\mathrm{C}-\mathrm{D})$ of incubation, respectively. PTZ-induced seizure-like behavior is expressed as mean actinteg units/5 min ( \pm SEM) during the 30-min recording period (A, C) and over 5-min time intervals (B, D). Means are pooled from three independent experiments with each 9-12 replicate wells per condition. Statistical analysis: (A, C) one-way ANOVA with Dunnett's multiple comparison test, (B, D) two-way ANOVA with Bonferroni posttests (GraphPad Prism 5). Significance levels: $* \mathrm{p} \leq 0.05 ; * * \mathrm{p} \leq 0.01 ; * * * \mathrm{p} \leq 0.001$. Abbreviation: VHC, vehicle.

Figure 4. Electrophysiological antiseizure analysis of azaspirofuran $A$ and pseurotin $A_{2}$ in the zebrafish PTZ seizure model. Noninvasive local field potential recordings from the optic tectum of larvae pre-exposed to vehicle (VHC) and pentylenetetrazole (PTZ), VHC only, compound and PTZ, or compound and VHC. (A-C) Larvae were incubated with $12.5 \mu \mathrm{g} / \mathrm{mL}$ azaspirofuran A for $2 \mathrm{~h}$, (D-F) or with $12.5 \mu \mathrm{g} / \mathrm{mL}$ pseurotin $\mathrm{A}_{2}$ for $18 \mathrm{~h}$, conform with the optimal condition used in the behavioral assay. (A, D) Larvae are considered to possess epileptiform brain activity when three or more events occurred during a 10-min recording. Epileptiform discharges are quantified by the number (mean $\pm \mathrm{SD})(\mathrm{B}, \mathrm{E})$ and cumulative 
duration (mean $\pm \mathrm{SD})(\mathrm{C}, \mathrm{F})$ of events per 10-min recording. Number of replicate wells per condition: $\mathrm{n}=33(\mathrm{~A}-\mathrm{C}$ ) and $\mathrm{n}=22(\mathrm{D}-\mathrm{F})$ for VHC + PTZ controls, $\mathrm{n}=31(\mathrm{~A}-\mathrm{C})$ and $\mathrm{n}=21$ (D-F) for VHC + VHC controls, $\mathrm{n}=15$ (A-C) and $\mathrm{n}=19$ (D-F) for compound + PTZ conditions, and $n=15$ (A-C) and $n=19$ (D-F) for compound + VHC conditions. Statistical analysis: (A, D) Fisher's exact test with Bonferroni posttest, (B-C, E-F) Kruskal-Wallis test with Dunn's multiple comparison test (GraphPad Prism 5). Significance levels: * $\mathrm{p} \leq 0.05$; ** $\mathrm{p} \leq 0.01$; *** $\mathrm{p} \leq 0.001$.

Figure 5. Representative local field potential recordings. 10-min noninvasive local field potential recordings from the optic tectum of larvae pre-exposed to vehicle (VHC) and pentylenetetrazole (PTZ), VHC only, compound and PTZ, or compound and VHC. Larvae were incubated with $12.5 \mu \mathrm{g} / \mathrm{mL}$ azaspirofuran A for $2 \mathrm{~h}$ or $12.5 \mu \mathrm{g} / \mathrm{mL}$ pseurotin $\mathrm{A}_{2}$ for $18 \mathrm{~h}$, conform with the optimal condition used in the behavioral assay.

Figure 6. Antiseizure activity analysis of azaspirofuran $A$ and pseurotin $A_{2}$ in the mouse 6-Hz (44 mA) psychomotor seizure model. Psychomotor seizures were electrically induced 30 min after i.p. injection of vehicle (VHC, $n=10)$, positive control valproate $(n=6)$, or test compound ( $\mathrm{n}=6-7)$, and 120 min after i.p. injection of negative control phenytoin $(n=5)$. Seizures are quantified by the duration (mean $\pm \mathrm{SD}$ ). Statistical analysis: one-way ANOVA with Dunnett's multiple comparison test (GraphPad Prism 5). Significance levels: $* \mathrm{p} \leq 0.05$; $* * \mathrm{p} \leq 0.01 ; * * * \mathrm{p} \leq 0.001$.

\section{TABLE LEGENDS}

Table 1. Maximum tolerated concentrations (MTCs) of test compounds in 7-dpf zebrafish larvae. In case no MTC was reached, $100 \mu \mathrm{g} / \mathrm{mL}$ was used as the test concentration.

Table 2. ADMET analysis of azaspirofuran A and pseurotin A2. Abbreviations: concentration at which an assay is inhibited by $50 \%$, IC50. 


\section{FIGURES}

\section{Figure 1.}

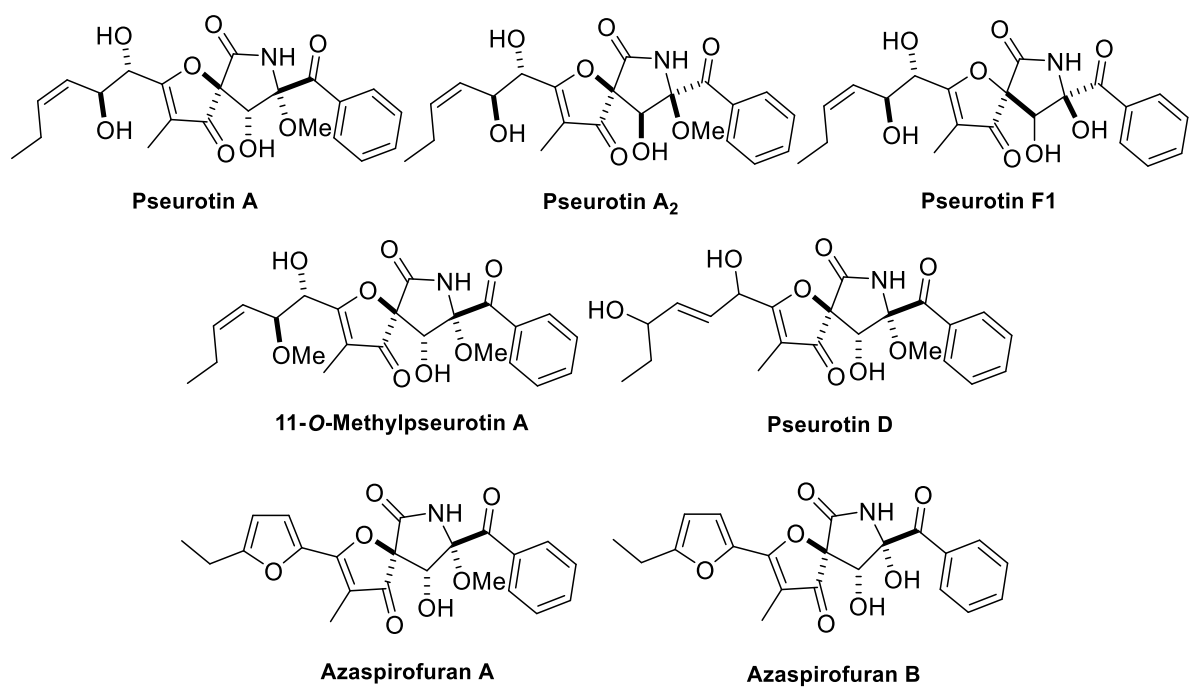

Figure 2.

A

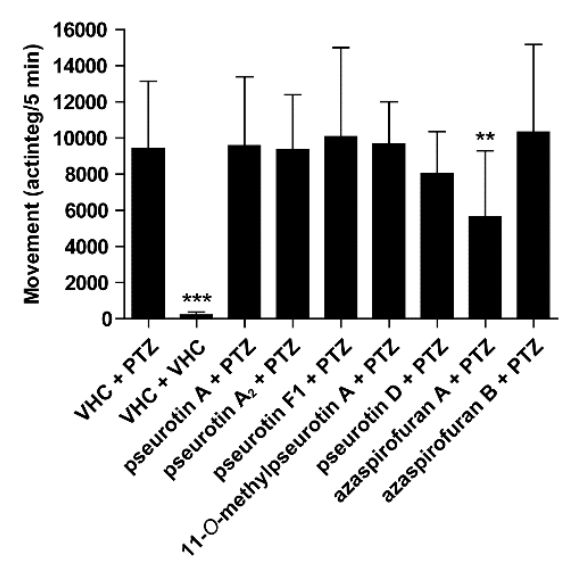

B

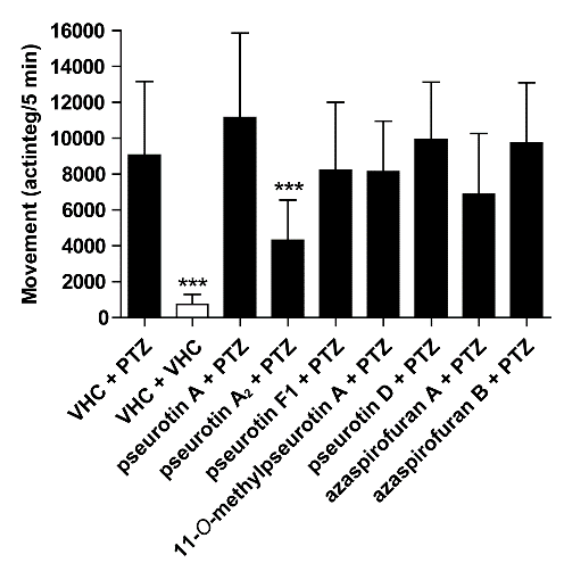


Figure 3.

A

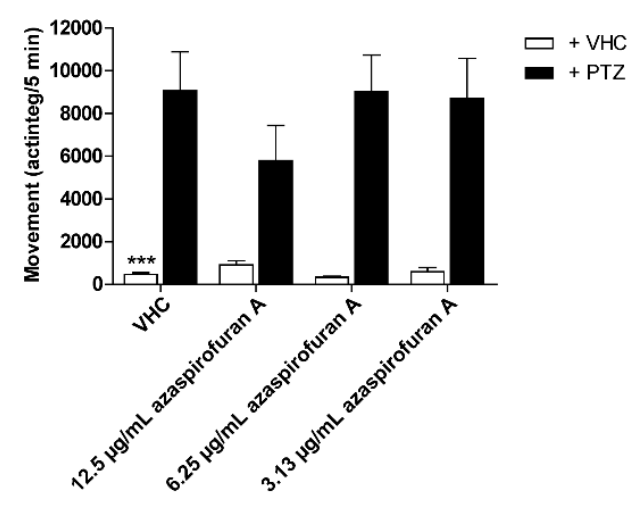

C

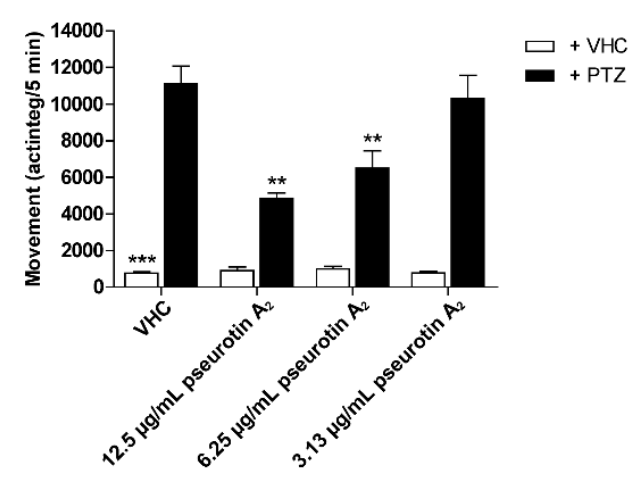

B

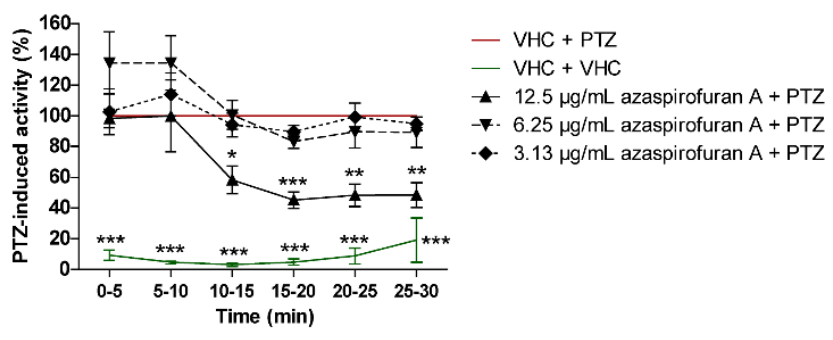

D

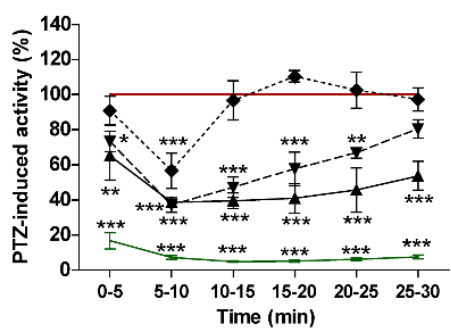

- $\mathrm{VHC}+\mathrm{PTZ}$

- $\mathrm{VHC}+\mathrm{VHC}$

\ $12.5 \mu \mathrm{g} / \mathrm{mL}$ pseurotin $A_{2}+$ PTZ

- $6.25 \mu \mathrm{g} / \mathrm{mL}$ pseurotin $A_{2}+$ PTZ

- $3.13 \mu \mathrm{g} / \mathrm{mL}$ pseurotin $A_{2}+P T Z$ 
Figure 4.

A

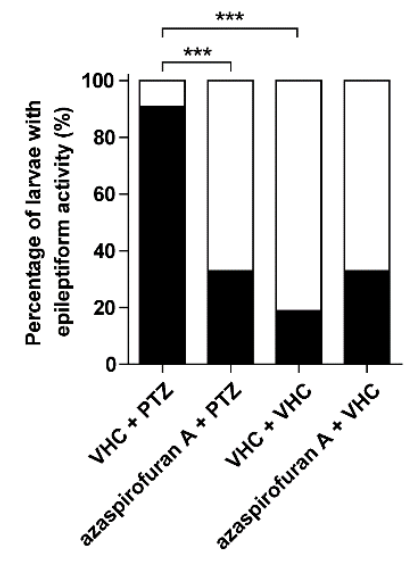

D

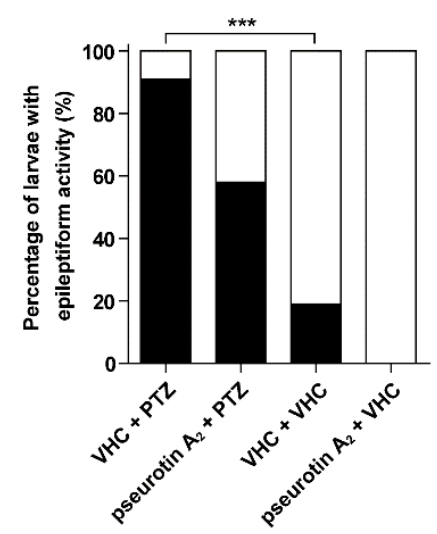

B

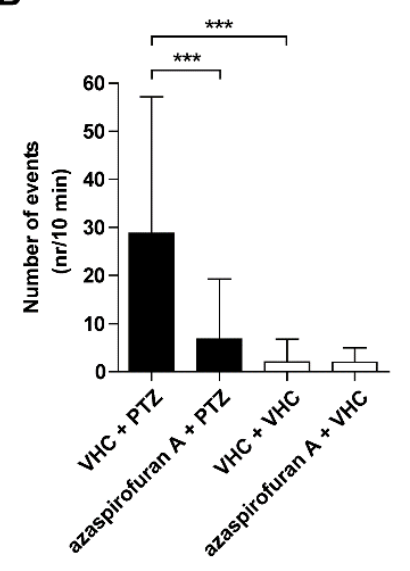

E

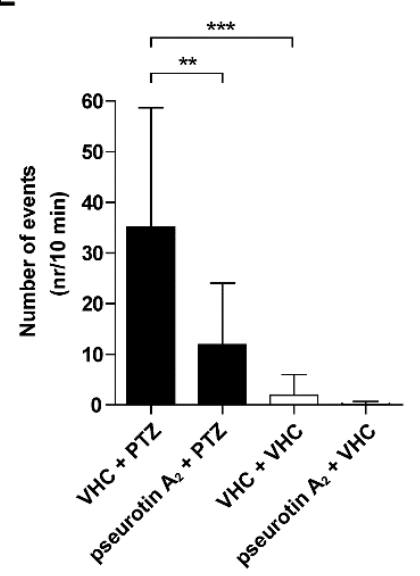

C

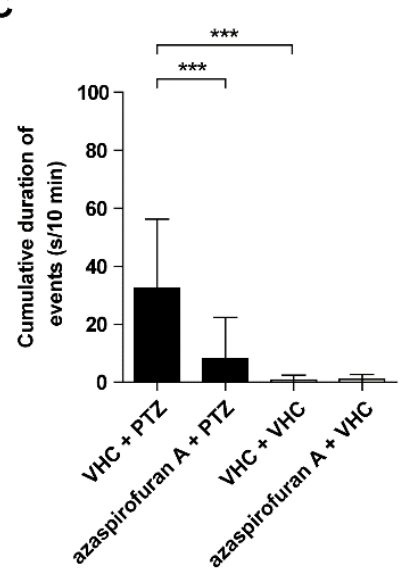

F

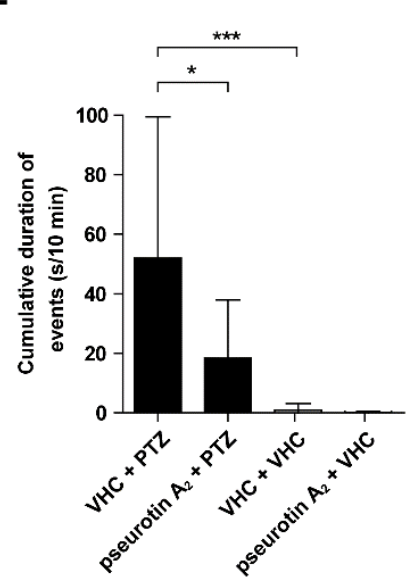


Figure 5.

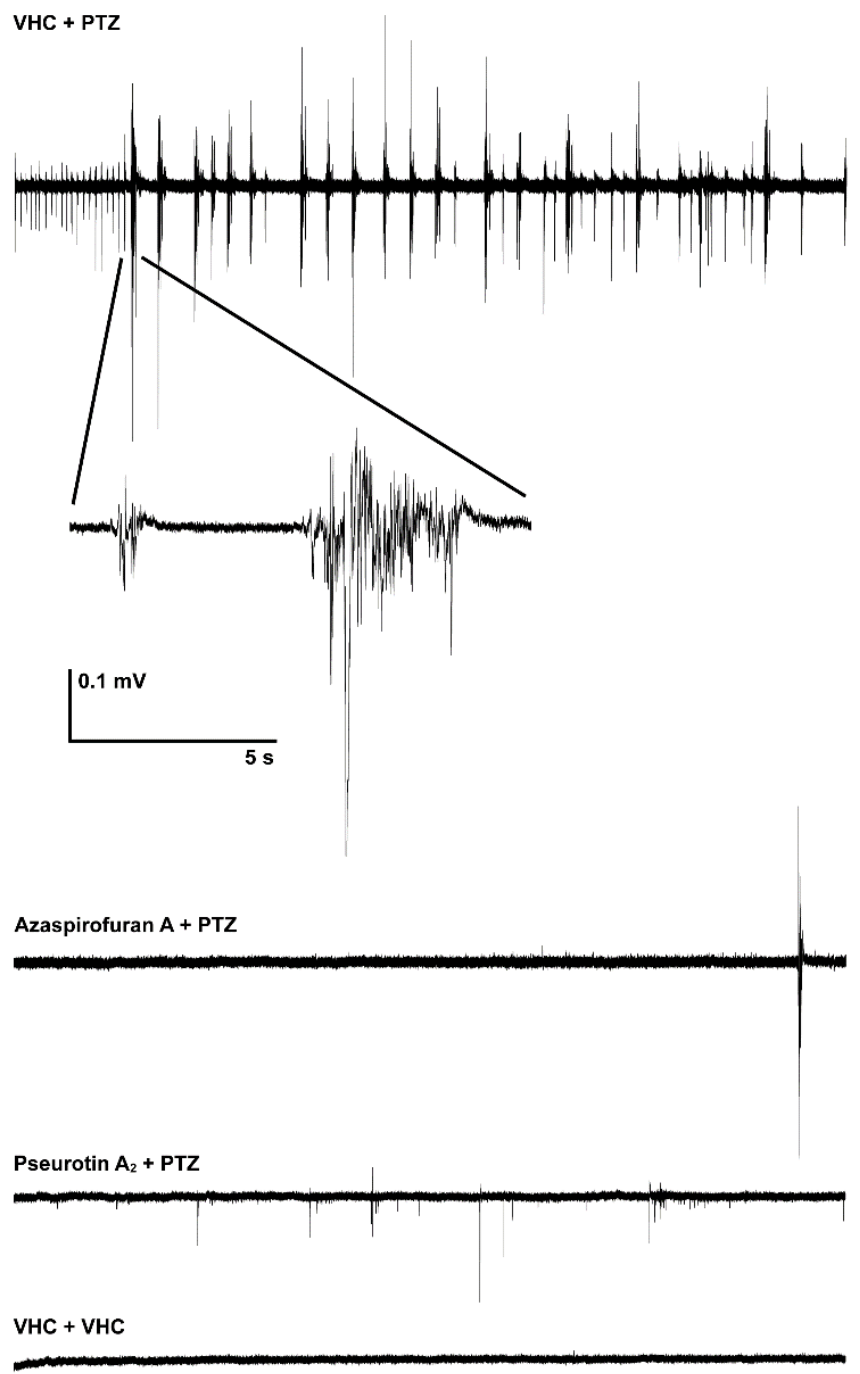

Azaspirofuran A + VHC

Pseurotin $\mathrm{A}_{2}+\mathrm{VHC}$

P

$0.1 \mathrm{mV}$

$2 \min$ 
Figure 6.

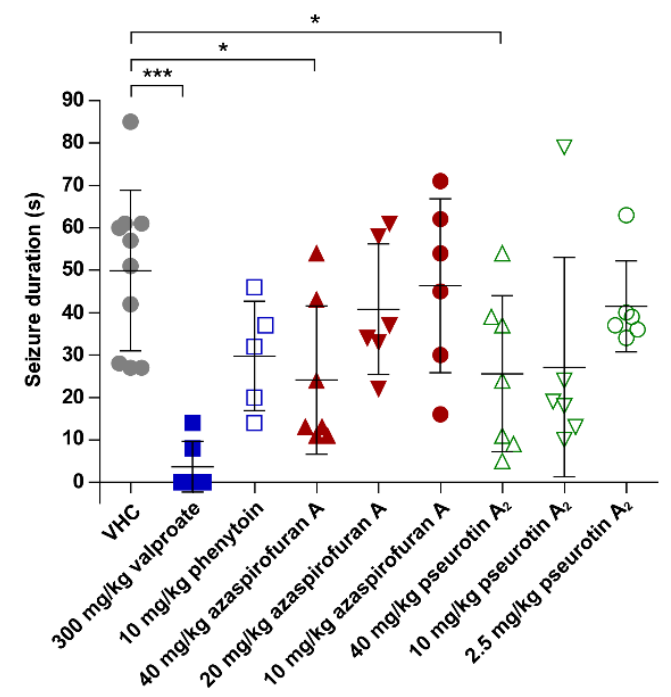

TABLES

Table 1.

\begin{tabular}{l|c}
\hline \multicolumn{1}{c|}{ Compound } & MTC $(\boldsymbol{\mu g} / \mathbf{m L})$ \\
\hline Pseurotin A & 100 \\
Pseurotin A2 & 12.5 \\
Pseurotin F1 & 50 \\
11-O-methylpseurotin A & 100 \\
Pseurotin D & 100 \\
Azaspirofuran A & 12.5 \\
Aaspirofuran B & 12.5 \\
\hline
\end{tabular}


Table 2.

\begin{tabular}{|c|c|c|c|c|}
\hline ADMET test & \multicolumn{2}{|c|}{ Azaspirofuran A } & \multicolumn{2}{|c|}{ Pseurotin $\mathbf{A}_{2}$} \\
\hline \multicolumn{5}{|c|}{ Cytotoxicity } \\
\hline HEPG2 cells & $\mathrm{IC}_{50}>50 \mu \mathrm{M}$ & No effect & $\mathrm{IC}_{50}>50 \mu \mathrm{M}$ & No effect \\
\hline THLE2 cells & $\mathrm{IC}_{50}>50 \mu \mathrm{M}$ & Weak decrease & $\mathrm{IC}_{50}>50 \mu \mathrm{M}$ & Weak decrease \\
\hline SHSY5Y cells & $\mathrm{IC}_{50}>50 \mu \mathrm{M}$ & Weak decrease & $\mathrm{IC}_{50}=17.9 \mu \mathrm{M}$ & Decrease \\
\hline \multicolumn{5}{|c|}{ Cardiotoxicity } \\
\hline Nav1.5 channel & $\mathrm{IC}_{50}=39.06 \mu \mathrm{M}$ & Low inhibitory effect & $\mathrm{IC}_{50}>50 \mu \mathrm{M}$ & No effect \\
\hline Cav1.2 channel & $\mathrm{IC}_{50}>50 \mu \mathrm{M}$ & No effect & $\mathrm{IC}_{50}>50 \mu \mathrm{M}$ & No effect \\
\hline hERG channel & $\mathrm{IC}_{50}>50 \mu \mathrm{M}$ & No effect & $\mathrm{IC}_{50}>50 \mu \mathrm{M}$ & No effect \\
\hline \multicolumn{5}{|c|}{ CYP450 enzymes } \\
\hline CYP3A4 & $\mathrm{IC}_{50}>88 \mu \mathrm{M}$ & No effect & $\mathrm{IC}_{50}>88 \mu \mathrm{M}$ & No effect \\
\hline CYP2D6 & $\mathrm{IC}_{50}=23.4 \mu \mathrm{M}$ & Weak inhibition & $\mathrm{IC}_{50}>88 \mu \mathrm{M}$ & No effect \\
\hline CYP2C9 & $\mathrm{IC}_{50}=47.3 \mu \mathrm{M}$ & Weak inhibition & $\mathrm{IC}_{50}>88 \mu \mathrm{M}$ & No effect \\
\hline \multicolumn{5}{|c|}{ Other factors } \\
\hline Hepatic clearance & $\begin{array}{l}20.17 \mu \mathrm{L} / \mathrm{min} / \mathrm{mg} \\
\text { protein }\left(\mathrm{t}_{1 / 2}=33.12\right. \\
\min )\end{array}$ & Medium & $\begin{array}{l}<8.6 \mu \mathrm{L} / \mathrm{min} / \mathrm{mg} \\
\text { protein }\left(\mathrm{t}_{1 / 2}>60\right. \\
\min )\end{array}$ & Low \\
\hline Kinetic solubility & $>100 \mu \mathrm{M}$ & Acceptable & $>100 \mu \mathrm{M}$ & Acceptable \\
\hline Protein binding & $95.42 \%$ & $\begin{array}{l}\text { Low percentage of free } \\
\text { drug; } 66.7 \% \text { recovery }\end{array}$ & $36.53 \%$ & $\begin{array}{l}\text { High percentage of free } \\
\text { drug; } 100 \% \text { recovery }\end{array}$ \\
\hline Permeability & $18.64 \times 10^{-6} \mathrm{~cm} \mathrm{~s}^{-1}$ & High & $0.05 \times 10^{-6} \mathrm{~cm} \mathrm{~s}^{-1}$ & Low \\
\hline
\end{tabular}


FOR TABLE OF CONTENTS USE ONLY

\section{Manuscript title}

Zebrafish-based discovery of antiseizure compounds from the Red Sea: pseurotin A2 and azaspirofuran $\mathrm{A}$

\section{Authors}

Daniëlle Copmans, Mostafa Rateb, Jioji N. Tabudravu, Mercedes Pérez-Bonilla, Nina Dirkx, Riccardo Vallorani, Caridad Diaz, José Pérez del Palacio, Alan J. Smith, Rainer Ebel, Fernando Reyes, Marcel Jaspars, Peter A. M. de Witte

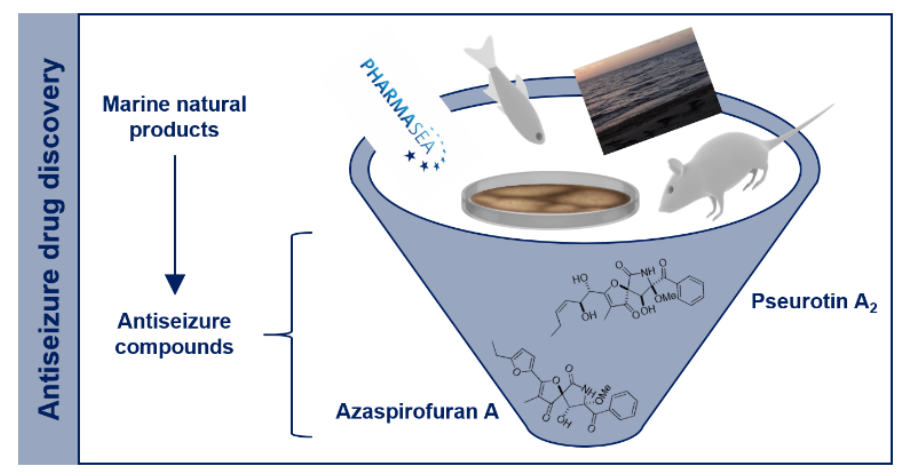

